\title{
Palets et minches de Gargantua. Mégalithismes dans le Centre-Ouest de la France
}

\section{Charles-Tanguy Le Roux}

\section{(2) OpenEdition}

12 Journals

Édition électronique

URL : http://journals.openedition.org/rao/5692

DOI : $10.4000 /$ rao. 5692

ISSN : $1775-3732$

Éditeur

Presses universitaires de Rennes

\section{Édition imprimée}

Date de publication : 31 décembre 2018

Pagination : 279-280

ISBN : 978-2-7535-8013-8

ISSN : 0767-709X

\section{Référence électronique}

Charles-Tanguy Le Roux, « Palets et minches de Gargantua. Mégalithismes dans le Centre-Ouest de la France », Revue archéologique de l'Ouest [En ligne], 35 | 2018, mis en ligne le 12 février 2020, consulté le 27 novembre 2020. URL : http://journals.openedition.org/rao/5692 ; DOI : https://doi.org/10.4000/rao. 5692 


\section{Analyses d'ouvrages}

Joussaume R, 2016 - Palets et minches de Gargantua. Mégalithismes dans le Centre-Ouest de la Fance. Chauvigny (Vienne), Association des Publications chauvinoises, 2016, 390 p. coll. "Memoria momenti: 39 ", ISSN 1294-5870, ISBN 979-1090534-39-1 (20,00€).

Ce $39^{\mathrm{e}}$ volume de la collection "Memoria momenti" est un bel ouvrage de plus à mettre à l'actif des productions de l'APC. Sous une agréable couverture cartonnée, Roger Joussaume nous propose, en 390 pages au format $17 \times 24 \mathrm{~cm}$ et avec un maquettage soigné, un panorama du phénomène mégalithique dans cette vaste région qui va de la Loire moyenne à la Gironde et de l'Atlantique au Limousin. Le papier couché permet une très belle qualité d'illustration (120 " figures " dont beaucoup sont en fait des planches composées rassemblant plusieurs documents - photos, plans et dessins d'objets).

Après les classiques préliminaires et avant une brève conclusion suivie d'une copieuse bibliographie (quelque 200 titres), le cœur du volume s'articule en deux « chapitres": I - Les monuments et II - Synthèse. En fait, il s'agit plutôt de "parties ", elles-mêmes subdivisées en douze sous-chapitres pour la première, six pour la seconde.

Compte tenu de l'importante façade maritime de la région étudiée et de la configuration de son littoral, on ne s'étonnera pas que ce premier " chapitre " soit précédé, sur une double page, par une carte où J.-M. Large a su visualiser avec talent - et malgré les aléas inhérents à l'exercice - une esquisse de ce qu'aurait pu être le littoral au Néolithique entre le sud de la Bretagne et la Gironde. On pourra regretter que cette carte ne descende pas un peu plus bas que Royan, et surtout que le diagramme chronologique associé soit calé sur le zéro NGF alors que la carte l'est sur celui des cartes marines. Il est vrai que la corrélation entre les deux est délicate : le décalage, lié à " l'établissement " de chaque port, est en moyenne d'environ $5 \mathrm{~m}$ sur la côte atlantique française, et donc proche du décalage estimé entre le niveau marin vers -4000 et l'actuel, ce qui ne simplifie pas les choses).

Chacun des sous-chapitres de cette première partie couvre une des douze entités territoriales annoncées dans la "présentation " et explicitées en fig. 3. Ce parti-pris d'un " tour de l'Ouest " géographique plutôt que d'entrées typologiques peut surprendre de prime abord; il est justifié par l'extrême diversité du patrimoine mégalithique au sein de cette vaste région (près de $300 \mathrm{~km}$ d'ouest en est et presque autant $\mathrm{du}$ nord au sud). Le prix à payer est - parfois - une certaine redondance interrégionale mais l'indéniable avantage est de nous offrir une série de " quasi-monographies " régionales, ce qu'il est bien agréable - et fort pratique - de trouver au sein d'un même ouvrage.

Au fil des pages, retenons tout d'abord l'impression d'un global déficit d'information sur ce patrimoine quant on le compare à une région il est vrai privilégiée comme la Bretagne. Heureusement, nombre de travaux récents (dont au premier rang ceux de l'auteur) ont, en quelques décennies, rassemblé un corpus d'information impressionnant en passe, dans certains domaines, d'égaler, voire parfois surpasser celui disponible pour la péninsule voisine. C'est aussi l'occasion de saluer les recherches en cours (dont le PCR "Monumentalismes et territoires entre Loire et Charente" dirigé par Vincent Ard), en plus des fouilles désormais emblématiques du "tumulus géant " de Prissé-la-Charrière près de Niort.

Dans le domaine des tombes à couloir avec espace sépulcral complexe, ce travail est l'occasion de souligner le vaste domaine allant du sud-ouest du Finistère à l'Anjou d'une part et à la forêt charentaise de la Boixe d'autre part, avec des expressions très variées terroir par terroir. Il en serait de même pour les pierres dressées - menhirs et stèles - dont les alignements s'expriment bien différemment selon que l'on est dans le sud-vendéen ou dans le Morbihan intérieur pour ne prendre que deux régions récemment documentées.

Depuis leur définition par le Dr Gruet, les « dolmens angevins constituent un véritable " type-symbole " du mégalithisme funéraire dans le Centre-Ouest et, grâce à son balayage territorial, Roger Joussaume nous en précise la zone d'extension, les relations avec d'autres architectures et - malgré un persistant déficit d'information en ce domaine - il propose quelques arguments de datation susceptibles de les faire remonter au Néolithique moyen. En ce qui concerne l'identification du type architectural sur des sites dégradés ou peu clairs, nous proposerons un critère sinon discriminant du moins susceptible de conduire à une certaine présomption; il s'agit de la dalle de chevet de la chambre, qui semble s'appuyer sur les parois latérales (et non l'inverse comme c'est généralement le cas dans les allées-couvertes). Tantôt flagrant tantôt discret, ce caractère témoigne en effet d'une 
conduite de chantier bien particulière et donc d'un savoirfaire spécifique.

Un autre élément d'architecture sur lequel Roger Joussaume insiste à juste titre, c'est la notion de « couloir non couvert " permettant d'approcher de la tombe avant de s'enfoncer dans les profondeurs du cairn. Un tel dispositif suppose cependant un monument relativement bas, au moins en périphérie, ce qui est loin d'être toujours le cas (pensons aux façades d'un seul jet reconnues à Carn ou Gavrinis). On saura enfin gré à l'auteur d'avoir élargi son sujet aux évidences de monumentalisme présumé funéraire bien que non mégalithique. Ce sont par exemple ces énigmatiques enclos de "type Passy " qui, après la Bourgogne, sont désormais bien attestés en Normandie et semblent avoir essaimé jusqu'en Poitou.

Le grand chapitre II nous propose une "synthèse du mégalithisme du Centre-Ouest de la France ». C'est là que Roger Joussaume nous propose ses réflexions transversales à partir des corpus présentés dans les pages qui ont précédé. Sont ainsi passées en revue les architectures, domaine où l'auteur distingue cinq types de dolmens à couloir et propose un phasage ternaire (4800-4500, 4500-4300, 4300-4000). Viennent ensuite les "premiers occupants des tombes ", un domaine où les fouilleurs en milieu non calcaire se sentent bien frustrés (en témoigne par exemple la fig. 135 rappelant toute l'information qui a pu être recueillie dans la tombe F0 de Bougon). Le matériel archéologique est ensuite passé en revue, avec quelques "vaisseliers " présentés côte-à-côte d'une manière fort pédagogique. Quant aux manifestations de ce que l'on appelle couramment "art mégalithique ", leur présentation confirme que, de Dissignac à la Boixe, le Centre-Ouest est loin d'être un désert. Un petit regret : que la présentation des récentes découvertes de la Bretellière n'ait pas été l'occasion de tordre le cou plus vigoureusement à ce que nous appellerons le " mythe du Cachalot ", apparu voici un quart de siècle en réaction à la vieille " hache-charrue » de Le Rouzic : ni l'une ni l'autre expression ne peuvent convenir, la première pour des raisons anatomiques (les cétacés ont perdu leur mobilité céphalique et ne peuvent donc avoir inspiré un signe coudé)), la seconde pour des raisons techniques (un soc ne peut travailler latéralement). Pour le signe en question, le plus sage ne serait-il pas de se contenter d'un "mot-code » à la manière de l'Objet des statues-menhirs du Midi ou de la Thing de la péninsule ibérique?

Tout cela est sans doute à prendre, au moins pour partie, comme un "état de la question " face aux recherches émergentes évoquées plus haut. Mais que cela ne décourage surtout pas les spécialistes - et les autres - de se plonger dans la belle synthèse de Roger Joussaume. On y aura plaisir à retrouver un spécialiste internationalement reconnu qui, une fois de plus, a su ne pas oublier le pédagogue qu’il fut dans une vie antérieure pour nous présenter son sujet d'une manière claire, agréable et accessible en s'appuyant sur une illustration abondante et de qualité.

Charles-Tanguy Le Roux

Nicolas C., 2016 - Flèches de pouvoir à l'aube de la métallurgie, de la Bretagne au Danemark (2500-1700 av.n. è.), Sidestone Press, Leiden, 2 vol., 430 et 520 p.

Cette publication de C. Nicolas aborde la période de transition entre la fin du Néolithique, le Campaniforme et le début de l'âge du Bronze en privilégiant le mobilier découvert en contextes funéraires, et plus particulièrement les armatures de flèches. Elle est directement issue de sa thèse de doctorat et très proche de la version de soutenance validée par le jury de thèse. Elle est organisée en deux volumes.

Le premier volume, que l'on pourrait qualifier de « texte illustré ", comprend les développements qui composent le corps principal de la thèse. Il est structuré selon un plan relativement classique et fluide : après une grosse introduction qui fixe le cadre théorique général de la thèse, et un bref exposé de la méthodologie employée, l'auteur explore différents axes d'ordre socio-économique à partir des pratiques funéraires et du mobilier d'accompagnement des défunts : gestion des productions et spécialisation artisanale, chrono- logie et typologie des flèches, inégalités sociales et identités culturelles, territorialité, circulation des objets et des idées, etc.

Le second volume, "catalogue ", expose la totalité du corpus sur lequel se fondent les développements du premier volume, sous la forme de fiches signalétiques établies pour chaque site. On y trouve à chaque fois les informations relatives à la localisation des sites, les dates et auteurs des fouilles, la quantité et la nature du mobilier découvert, les datations absolues et les principales ressources bibliographiques pour chaque site. Les fiches signalétiques sont complétées par l'iconographie la plus complète possible pour ces sites, lorsque c'est utile : plan des structures archéologiques, photographies, planches de mobilier. Les sites du corpus sont regroupés par région d'étude, indexés et localisés sur des cartes, ce qui facilite l'accès à tout type d'information. 